\title{
Procesos de enseñanza y de aprendizaje del número relativo en contextos de la vida cotidiana (ascensos-descensos, perdidas-ganancias) teniendo en cuenta las diferentes operaciones básicas y su respectiva representación en la recta numérica ${ }^{1}$
}

\author{
Ensinar e aprender no número relativo em contextos da vida quotidiana \\ (promoções, rebaixamentos,-lucros cessantes), tendo em conta as diferentes \\ operações básicas e sua respectiva representação em numéricaón reta.
}

Ensinar e aprender no número relativo em contextos da vida quotidiana (promoções, rebaixamentos,-lucros cessantes), tendo em conta as diferentes operações básicas e sua respectiva representação em numéricaón reta.no

Recibido: mayo 2013

Aceptado: agosto 2013
Diego Medina Sánchez ${ }^{2}$ Karen Liliana Farfán Rincón ${ }^{3}$ Diego Alejandro Villalba Giraldo ${ }^{4}$

\section{Resumen}

El siguiente documento da cuenta de los procesos de enseñanza y de aprendizaje que se llevaron a cabo en la Institución Educativa Distrital Santa Martha con estudiantes de grado séptimo para la enseñanza del número relativo en contextos de la vida cotidiana utilizando "ascensos-descensos, perdidas-ganancias" para luego llevarlo a la representación en la recta numérica y las distintas operaciones con estos números.

Para la enseñanza del numero relativo se toman unas fases que presentan (Vargas, I. y otros, 1990), y teniendo en cuenta las fases, se propone trabajar las primeras con una serie de situaciones contextuales, para luego ir llevándolo a campos más abstractos en las últimas etapas, y que por último los estudiantes reconozcan la utilidad del numero relativo y sus aplicaciones.

Palabras clave: Matemáticas escolares; números; operaciones aritméticas; números relativos; transposición didáctica; representación en la recta numérica; operaciones; fases de aprendizaje.

1 Artículo de Investigación

2 Universidad Distrital Francisco José de Caldas. Bogotá, Colombia. Contacto: dms239@hotmail.com

3 Universidad Distrital Francisco José de Caldas. Bogotá, Colombia. Contacto: liana0206@hotmail.com.

4 Universidad Distrital Francisco José de Caldas. Bogotá, Colombia. Contacto: diegosanta-19@hotmail.com 


\section{Abstract}

This paper gives an account of the processes of teaching and learning that took place in the District Educational Institution Santa Martha with seventh grade students for teaching relative numbers in everyday life contexts using "promotions, demotions, loss-profits "and then take the representation on the number line and the various operations with these numbers.

For teaching the relative number taken some steps that have (Vargas, I. et al, 1990), and taking into account the phases, the first plans to work with a number of contextual situations, then go taking it to more abstract fields in the latter stages, and lastly students to recognize the usefulness of relative number and their applications.

Keywords: School Mathematics, numbers, arithmetic, integers, didactic transposition; representation on the number line; operations; learning phases.

\section{Resumo}

O artigo apresenta os resultados parciais de uma investigação na qual Este artigo dá conta dos processos de ensino e aprendizagem que ocorreram no Distrito Instituição Educacional Santa Martha com alunos do sétimo para o ensino de números relativos em contextos da vida quotidiana usando "promoções, rebaixamentos, perda de lucros "e, em seguida, tomar a representação sobre o número da linha e as várias operações com esses números.

Para ensinar o número relativo tomado algumas medidas que têm (Vargas, I. et al, 1990), e tendo em conta as fases, os primeiros planos para trabalhar com uma série de situações contextuais, então vá levá-lo para os campos mais abstratos nas últimas fases, e, por último estudantes para reconhecer o número relativo de utilidade e as suas aplicações.

Palavras-chave: Escola Matemática, números, aritmética, inteiros, transposição didática; representação na linha de número; operações; fases de aprendizagem.

\section{Contextualización}

Para la planeación de las clases se utilizó el modelo DECA el cual sintetiza una forma de trabajar durante todas las sesiones de clase y de igual manera la forma de trabajar en cada una de ellas. Inicia con una prueba diagnóstico para identificar los conocimientos previos que tienen los estudiantes sobre el concepto a enseñar, luego se realiza una serie de actividades para iniciar a los estudiantes en dicho concepto, de acuerdo a estos primeros procesos se procede a formalizar y por último se evalúa.

En las primeras actividades se logra que los estudiantes caigan en un conflicto cognitivo para poder forzar los cambios de los esquemas mentales que ya tienen con respecto al número relativo, para luego poder ver como dice Gonzales (1991) la relación útil y la relación como objeto y así poder evidenciar con ayuda de la recta numérica una introducción al cero relativo. 


\section{Referentes teórico-prácticos}

En el presente trabajo se abordará el concepto de los números relativos, los cuales pertenecen al pensamiento numérico. Estos números son el resultado de la relación de dos números, generalmente la división de uno por el otro. Los números relativos han surgido como una cuestión de conveniencia, principalmente con el objetivo de poder representar adecuadamente las magnitudes cuyas cantidades son susceptibles de ser agrupadas en dos categorías, o de ser consideradas en sentidos opuestos. Son muchas las magnitudes de este tipo, llamadas magnitudes relativas que se representan en la vida cotidiana, en el campo de la Física y en otras disciplinas científicas.

Según Torres (2007) Los números negativos, extienden el conjunto de los números naturales, formando los números enteros: la generalidad para la operación de resta y división. Por ejemplo 5 - 9 resulta -4 , que no es natural, donde no se cumple entonces la propiedad de cerradura (operaciones que se realizan dentro de un mismo conjunto numérico) en los naturales.

Se realiza un trabajo con números y operaciones, que generan el desarrollo de habilidades y destrezas numéricas, lo cual es reconocido por la SED (2007), pues asegura que: "la importancia no surge del aprendizaje de las definiciones y algoritmos, sino que se debe generar en el estudiante herramientas que permitan facilitar la comprensión y el actuar en situaciones que involucren los diferentes tipos de números" en este caso el conjunto numérico de los enteros $(Z)$, para dar una complejidad a las operaciones intelectuales.

Las investigaciones de González (1995) muestran que el orden que inducen los modelos concretos no es el de los números enteros: mientras en $\mathrm{Z}$ se define un orden total, los modelos concretos inducen dos órdenes parciales y opuestos referidos a las regiones positiva y negativa. Por estas y otras razones didácticas, se plantea la construcción de un nuevo objeto matemático, el 'número natural relativo'. Dicho número que, según el autor, refleja mejor el comportamiento de los modelos, ocuparía una posición intermedia entre el número natural y el entero. Así pues, según esta propuesta, el trabajo en la escuela con situaciones aditivas de comparación conduciría a la noción de 'número natural relativo' desde la cual, más adelante, habría que pasar a la de número entero.

Marco didáctico. Para los procesos de enseñanza y de aprendizaje se tiene en cuenta la teoría de la transposición didáctica, que para Chevallard (1998) es la transformación de un objeto de saber enseñar en un objeto de enseñanza; es la transición de conocimientos matemáticos a conocimientos escolares.

Para la enseñanza del número relativo se tienen en cuenta unas fases propuestas por (Vargas, I. y otros, 1990) las cuales son:

- El número relativo como relación en contextos concretos.

- De la relación-útil a la relación-objeto contextos concretos con el número natural implícito.

- El número relativo como objeto contextualizado.

- Del número relativo como objeto contextualizad.

- El número entero como útil matemático

- El número relativo como objeto contextualizado

Marco Matemático. Para la enseñanza del número relativo nos remontamos en un marco histórico, según dice González (1990) en su libro Número entero, la aparición de éstos es diferente a la de los números naturales o racionales debido a que su desarrollo no fue introducido por medio de magnitudes sino que tiene su origen en manipulaciones algebraicas; en las que se proporcionan respuestas únicamente a las ecuaciones que tengan solución con este tipo de números, Ejemplo: el caso de .

Es importante observar el desarrollo que tomó el número relativo a través de la historia: el problema más importante que enfrentaron los números 
negativos fue tomar estos números como una iniciación intelectual, para dar solución a este problema se justificaron en las leyes lógicas y aritméticas.

\section{Descripción general de la experiencia}

Los jóvenes de grado séptimo del colegio Institución Educativa Distrital Santa Martha comprendieron en buena medida la temática del número relativo asociándolo a casos de la vida cotidiana como el comprar en la tienda o deber dinero entre otros aspectos.

El proceso que se llevo fue pertinente debido a las conexiones de unas actividades con otras y a que se seguía un circuito cuyo fin era que los estudiantes comprendieran el número relativo, este proceso hizo que a su vez los estudiantes aceptaran con gusto las actividades, y los motivara a trabajar de la mejor manera

Es importante mencionar la fundamentación teórica que se requirió para los procesos de enseñanza y de aprendizaje, pues se apropió la temática y se hizo la transposición didáctica para volver un conocimiento puro en un conocimiento para enseñar y tener en cuenta la fuerte estructura teórica que se tuvo; debidamente conectada para no tener mayores problemas a la hora de enseñar los números relativos.

\section{Logros y dificultades evidenciadas}

Se cumplieron los propósitos planteados entre los cuales el propósito prioritario era el de reconocer los conocimientos que lograron obtener los estudiantes después de las sesiones de clases trabajadas con la temática del número relativo.

Se logró en los procesos de enseñanza y de aprendizaje ubicar los problemas de números relativos en la recta numérica y hacer movimientos en ella, fortaleciendo la parte operacional, donde se evidencio su agilidad para poder hacer operaciones y darle coherencia a las respuestas de los problemas planteados.

Por otra parte con respecto a la norma medicional se les recuerda a los estudiantes el cuidado del material debido a que una de las dificultades era que no lo cuidaban y no podían comprender la temática a trabajar con el número relativo.

Los siguientes son los objetivos planteados y que se cumplieron a cabalidad para el docente y el estudiante en pro del desarrollo de la secuencia:

\section{Docente.}

- Diseñar actividades que den cuenta de la gestión e interacción en el aula y en la construcción de los conceptos a trabajar.

\section{Estudiante.}

- Realiza operaciones entre números enteros.

- Ubica correctamente cantidades en la recta numérica.

\section{Reflexión final}

Es importante tener una buena estructura de trabajo basada en un marco teórico que le dé la suficiente fuerza para la realización y planeación de actividades. Por otra parte la metodología de trabajo en grupo fue importante debido a que los estudiantes podían ver los procesos que realizaban sus otros compañeros, analizaban si estaban bien o mal y daban aportes importantes entre ellos, así mismo el material que se les proporciono a los estudiantes fue muy bueno debido a que se interesaban bastante en la clase y se les permitía desarrollar sus propios conocimientos matemáticos. Dichos conocimientos fueron adquiriéndolos de manera autónoma, se logró que con cada institucionalización quedaran conocimientos claros. Las socializaciones también fueron parte vital pues se podían observar varios procedimientos posibles, que luego podrían utilizar en otra actividad. 


\section{Referencias}

Alvarado, J., Charry, G. (2009) Manifestaciones de la devolución que hace el profesor en la comprensión que los estudiantes adquieren en torno a la operatividad de los números relativos (suma y resta) en el grado séptimo.

Gonzales, G., Iriarte, M., Jimeno. M., Ortiz, A., Sanz, E., Machuca. I. (1990) Números Enteros. Ed. Síntesis. España

GONZÁLEZ MARÍ, J.L. (1995), El campo conceptual de los números naturales relativos, tesis doctoral, Universidad de Granada.
Quintero, A., Puentes, J. Bohórquez, L., Méndez, N. (2011) El número relativo como objeto contextualizado por medio de situaciones (temperatura, cronología y alturas) y operar con estos nuevos números, Universidad Distrital Francisco José de Caldas, Bogotá.

Torres, C (SF). Números enteros: origen e historia. Disponible en red: http://personales.ya.com/ casanchi/mat/enteros01.pdf 